\title{
THE TRANSITIVE PROPERTY OF PARALLEL LINES IS A CHARACTERISTIC PROPERTY OF REAL STRICTLY CONVEX BANACH SPACES
}

\author{
J. E. VALENTINE
}

\begin{abstract}
In a recent paper Freese and Murphy said a complete, convex, externally convex metric space has the vertical angle property provided for each four of its distinct points $p, q, r, s$, if $m$ is a midpoint of $p$ and $q$ and of $r$ and $s$, then $p r=q s$. In this paper we say a line $L$ is parallel to a line $N$ in such a space provided $L$ and $N$ contain points $p, r$, and $q, s$, respectively, such that the segments $S(p, q)$ and $S(r, s)$ have a common midpoint $m$. We further assume that if line $L$ is parallel to line $N$ and line $N$ is parallel to line $R$, then $L$ is parallel to $R$. The main result of this paper is that such a space is a real strictly convex Banach space. Since real strictly convex Banach spaces have all of the above properties, the characterization is then complete.
\end{abstract}

The problem of characterizing Banach spaces among the class of complete, convex, externally convex metric spaces has been solved by various authors, see [1, 2 , 4, 6 and 7]. (It is well known that a complete, convex, externally convex metric space contains a (metric) line for each pair of its distinct points, see [3].) The purpose of this paper is to show that the transitive property of parallelism does this. Of course, before we can talk about the transitive property of parallel lines, we need some way of defining what it means to say (metric) lines are parallel in a metric space. The vertical angle property of Freese and Murphy [5] provides a way.

A metric space has the vertical angle property, VAP, provided for each four of its distinct points $p, q, r, s$, if $m$ is a midpoint of $p$ and $q$ and of $r$ and $s$, then $p r=q s$.

Let $M$ be a complete, convex, externally convex metric space with VAP. We will say a line $L_{1}$ is parallel to a line $L_{2}$ provided $L_{1}$ and $L_{2}$ contain points $p, r$ and $q, s$, respectively, such that the segments $S(p, q)$ and $S(r, s)$ have a common midpoint $m$. We will say that a metric space $M$ has the transitive property of parallel lines, TPPL, provided if $L_{1}$ is parallel to $L_{2}$ and $L_{2}$ is parallel to $L_{3}$, then $L_{1}$ is parallel to $L_{3}$.

Throughout the remainder of this paper, $M$ will denote a complete, convex, externally convex metric space that has VAP and TPPL. Our first theorem is proved in [5], but we include a proof here in order to make the paper complete.

THEOREM 1. Each two distinct points of $M$ lie on a unique metric line.

Received by the editors September 5, 1984 and, in revised form, February 1, 1985.

1980 Mathematics Subject Classification. Primary 51K05, 51F99.

Key words and phrases. Banach space, convex, externally convex, metric space, strictly convex, transitive property of parallel lines, vertical angle property. 
Proof. As noted above, it is well known that each pair of distinct points of $M$ lie on at least one metric line. If $M$ contains a pair of distinct points that lie on two distinct lines, then distinct points $p, m, s^{\prime}, q$ can be found such that $m$ is a midpoint of $p$ and $q$ and $m$ is a midpoint of $p$ and $s^{\prime}$. If a midpoint of $m$ and $s^{\prime}$ is not between $m$ and $q$, let $s$ be that midpoint of $m$ and $s^{\prime}$ and let $r$ be a midpoint of $p$ and $m$. It follows that $m$ is a midpoint of $p$ and $q, m$ is a midpoint of $r$ and $s$, but $p r \neq q s$, which contradicts that $M$ has VAP. If every midpoint of $m$ and $s^{\prime}$ is between $m$ and $q$, it is easily seen that a segment $S$ with endpoints $m$ and $s^{\prime}$ contains a point $x$ closest to $s^{\prime}$ that is between $m$ and $q$. If $s$ is a midpoint of $x$ and $s^{\prime}$ on $S$ and if $r$ is a point betwen $p$ and $m$ such that $m s=m r$, then we again arrive at the contradiction that $M$ has VAP.

THEOREM 2. If $L$ is a line of $M$ and $p$ is a point of $M, p$ not on $L$, then $M$ contains a line $N$ such that $p$ is on $N$ and $N$ is parallel to $L$.

Proof. Let $q$ and $s$ be distinct points on $L$ and let $m$ be the midpoint of $p$ and $q$. Now denote by $r$ the point on the line joining $m$ and $s$ such that $m$ is the midpoint of $r$ and $s$. By definition, the line $N$ that contains $p$ and $r$ is parallel to $L$.

THEOREM 3. If $L$ and $N$ are distinct lines of $M$ and if $L$ is parallel to $N$, then $L$ and $N$ have no common points.

Proof. If $L$ is parallel to $N$, then $L$ and $N$ contain points $p, r$ and $q, s$, respectively, such that the segments $S(p, q)$ and $S(r, s)$ have a common midpoint $m$. By means contradiction, suppose $L$ and $N$ have a common point $x$. If $y$ is the point such that $m$ is the midpoint of $x$ and $y$, then by VAP, $p y=x q$ and $x s=r y$, and since $x, q, s$ are collinear, so are $r, p, y$. Similarly, by VAP, $q y=x p$ and $x r=s y$ and $q, s, y$ are collinear. This says that $L$ and $N$ have two common points contrary to the fact that lines are unique, unless $m=x$. But if $m$ is on $L$ and $N$ it follows that $L=N$, contrary to the fact that $L$ and $N$ are distinct.

COROLlaRY 1. If $L$ is a line of $M$ and if $p$ is a point of $M, p$ not on $L$, then there is a unique line $N$ of $M$ such that $p$ is on $N$ and $N$ is parallel to $L$.

Proof. By Theorem 2 there is at least one line $N$ that contains $p$ and is parallel to $L$. If there were another line $N^{\prime}$ that contains $p$ and is parallel to $L$, then clearly $L$ is parallel to $N^{\prime}$ and, by TPPL, $N$ is parallel to $N^{\prime}$, which contradicts Theorem 3 .

LEMMA 1. If $m$ is the midpoint of $p$ and $q$ and of $r$ and $s$, if $m_{1}, m_{2}$ are the respective midpoints of $p$ and $r$ and $q$ and $s$, and if $m_{3}, m_{4}$ are the respective midpoints of $p$ and $s$ and $q$ and $r$, then $m$ is the midpoint of $m_{1}$ and $m_{2}$ and of $m_{3}$ and $m_{4}$.

Proof. Let $m^{\prime}$ be the point of $M$ such that $m$ is the midpoint of $m_{2}$ and $m^{\prime}$. By VAP, $p r=q s, q m_{2}=p m^{\prime}$, and $m_{2} s=m^{\prime} r$. Since by hypotheses $q m_{2}=m_{2} s=q s / 2$, we have $p m^{\prime}=m^{\prime} r=p r / 2$. But $m$ is the midpoint of $p$ and $r$ and, consequently, by Theorem 1, $m_{1}=m^{\prime}$. Similarly, $m$ is the midpoint of $m_{3}$ and $m_{4}$.

LEMMA 2. Let $p, q, r$ be noncollinear points of $M$, and let $p^{\prime}, q^{\prime}, r^{\prime}$ be the respective midpoints of $q$ and $r, r$ and $p$, and $p$ and $q$. If $s$ and $t$ are points of $M$ such that $q^{\prime}$ is the midpoint of $p^{\prime}$ and $s$ and $r^{\prime}$ is the midpoint of $p^{\prime}$ and $t$, then $p$ is the midpoint of $s$ and $t$. 
Proof. Let $p, q, r, p^{\prime}, q^{\prime}, r^{\prime}, s, t$ denote points which satisfy the conditions of the lemma. Since $q^{\prime}$ is the midpoint of $p$ and $r$ and of $p^{\prime}$ and $s$, the line $\mathrm{L}(p, s)$ is parallel to the line $\mathrm{L}(q, r)$ by definition of parallel lines. Similarly, since $r^{\prime}$ is the midpoint of $p^{\prime}$ and $t$ and of $p$ and $q$, the line $\mathrm{L}(p, t)$ is parallel to $\mathrm{L}(q, r)$. Since $\mathrm{L}(p, s)$ and $\mathrm{L}(p, t)$ both contain $p$ and are parallel to $\mathrm{L}(q, r)$ by Corollary $1, \mathrm{~L}(p, s)=\mathrm{L}(p, t)$ and since $p s=q p^{\prime}=p^{\prime} r=t p$, it follows that $p$ is the midpoint of $s$ and $t$.

We are now ready to complete the characterization by appealing to a result of Andalafte, Valentine, and Wayment. In [2], they say a metric space has the Median Bisector Property, MBP, provided if $p, q, r$ is any noncollinear triple of points of the space and if $p^{\prime}, q^{\prime}, r^{\prime}$ are the midpoints of $q$ and $r, r$ and $p$, and $p$ and $q$, respectively, then any midpoint $t$ of $p$ and $p^{\prime}$ is a midpoint of $q^{\prime}$ and $r^{\prime}$ and, conversely, any midpoint of $q^{\prime}$ and $r^{\prime}$ is a midpoint of $p$ and $p^{\prime}$. They showed that a complete, convex, externally convex metric space is a real strictly convex Banach space if and only if it has the MBP.

THEOREM 4. The space $M$ has the MBP.

Proof. Let $p, q, r, p^{\prime}, q^{\prime}, r^{\prime}, s$ and $t$ satisfy the conditions of Lemma 2, and let $m$ denote the midpoint of $p$ and $p^{\prime}$. If $s^{*}$ and $t^{*}$ are points of $M$ such that $m$ is the midpoint of $q$ and $s^{*}$ and of $r$ and $t^{*}$, then it follows from the VAP that $p$ is the midpoint of $s^{*}$ and $t^{*}, \mathrm{~L}\left(s^{*}, t^{*}\right)$ is parallel to $\mathrm{L}(q, r)$, and consequently from Corollary 1 that $s^{*}=s$ and $t^{*}=t$. By Lemma $1, m$ is the midpoint of $q^{\prime}$ and $r^{\prime}$. Thus $M$ has the MBP.

THEOREM 5. A complete, convex, externally convex metric space is a real, strictly convex Banach space if and only if it has the VAP and the TPPL.

Proof. Theorem 4 and Theorem 2.1 of [2].

\section{REFERENCES}

1. E. Z. Andalafte and L. M. Blumenthal, Metric characterizations of Banach and Euclidean spaces. Fund. Math. 55 (1964), 23-55.

2. E. Z. Andalafte, J. E. Valentine and S. G. Wayment, Triangle median properties which characterize Banach spaces, Houston J. Math. 5 (1979), 307-312.

3. L. M. Blumenthal, Theory and applications of distance geometry, Oxford Univ. Press, London, 1953.

4. Raymond Freese and Grattan Murphy, The cmp and Banach spaces with unique metric lines, J. Geom. 14 (1980), 50-58.

5. Strictly convex spheres in metric spaces, Fund. Math. 117 (1983), 109-115.

6. J. E. Valentine, The Menelaus property characterizes real rotund Banach spaces, Fund. Math. 100 (1978), 41-44.

7. J. E. Valentine and S. G. Wayment, The Ceva property characterize real, strictly convex Banach spaces, Proc. Amer. Math. Soc. 78 (1980), 559-567.

Department of Mathematics, University of Texas at San antonio, San antonio, Texas 78285 